\title{
ATLAS upgrades for the SuperLHC collider
}

\section{Sandro Palestini}

CERN

1211 Geneva 23, Switzerland

E-mail: sandro.palestini@cern.ch

\section{On behalf of the ATLAS collaboration}

While LHC is getting ready for collisions, ATLAS is studying upgrades solutions necessary to cope with the scenarios of a three-fold increase in the collision rate, $3 \cdot 10^{34} \mathrm{~cm}^{-2} \mathrm{~s}^{-1}$, for phase-1 upgrade and of a ten-fold increase for the Super-LHC upgrade.

This contribution describes the expected experimental conditions and the main detector upgrade studies for ATLAS, which include an entirely new inner tracker system.

The detector technologies being considered for the upgrade of the Muon Spectrometer are discussed.

European Physical Society Europhysics Conference on High Energy Physics EPS-HEP 2009,

Krakow , Poland

July 16-22, 20 


\section{LHC plans: phase-1 and phase-2}

The program of repairs and safety measures for LHC is nearly completed, and the collider is expected to deliver beams before the end of 2009, and to continue in 2010 with a long run at a c.m. energy of $7 \mathrm{GeV}$ and luminosity up to $\sim 1 \cdot 10^{32} \mathrm{~cm}^{-2} \mathrm{~s}^{-1}$, for an integrated value of $\sim 100 \mathrm{pb}^{-1}$.

The road to high luminosity requires new beam elements, including the final-focusing triplets, and will benefit from the new Linac-4, currently under construction. The completion of this program defines the phase-1 upgrade, which will be achieved after a shut-down currently scheduled for 2013-2014, and will allow a peak luminosity of $3 \cdot 10^{34} \mathrm{~cm}^{-2} \mathrm{~s}^{-1}$, a factor of three higher than the original nominal luminosity of LHC. At this rate, at ATLAS or CMS the number interactions per beam crossing $(40 \mathrm{MHz})$ is equal to about 70 .

A second upgrade, called phase-2, is being designed with the aim of reaching a peak luminosity of $\sim 1 \cdot 10^{35} \mathrm{~cm}^{-2} \mathrm{~s}^{-1}$. This challenging machine upgrade [1] may affect the detectors in several significant ways:

- Interaction region components might be placed closer to the interaction points, requiring changes in the detectors and shielding configurations.

- The bunch spacing might be changed from 25 to $50 \mathrm{~ns}$ (the option of $12.5 \mathrm{~ns}$ is currently discarded for beams related reasons).

- The machine cycle and the luminosity variation during a fill will depend on the choice made for the design of the interaction regions. The different options include schemes with $\sim 300$ or $\sim 400$ interactions per crossing at peak luminosity.

\section{ATLAS upgrades: phase-1}

The ATLAS detector was designed for the original LHC peak luminosity of $1 \cdot 10^{34} \mathrm{~cm}^{-2} \mathrm{~s}^{-1}$, and studies have been undertaken on order to understand whether all subsystems would perform optimally with the three-fold luminosity increase expected for phase-1.

In particular, the performance of the innermost layer of the tracking system, part of the Pixel system, is expected to start degrading beyond $1 \cdot 10^{34} \mathrm{~cm}^{-2} \mathrm{~s}^{-1}$ and $\sim 700 \mathrm{pb}-1$ of peak and integrated luminosity, respectively. Both parameters will be exceeded in the phase-1 of LHC, and a program is underway for the installation of a new innermost pixel layer, the Insertable BLayer (IBL). The inner radius of the new detector will be at about $3.2 \mathrm{~cm}$ from the beam axis [2]. Different options are still being considered for the sensors, including planar-silicon, for which studies are under way for application in the more demanding regime of phase-2, and novel 3-D sensors [3] .

\section{ATLAS upgrades: phase-2}

The challenges of operating a detector in a hadron collider at a luminosity of about $1 \cdot 10^{35}$ $\mathrm{cm}^{-2} \mathrm{~s}^{-1}$ are of a different nature, including the performance and the stability of the various subdetectors, the performance of the trigger system and the data acquisition capability. Some main areas of study are discussed in the following sections. 


\subsection{New inner tracking detector}

ATLAS has been built with an inner tracking system formed by three layers of Silicon pixel detector, four double layers of silicon strip detectors, and a Transition Radiation Detector with thin tubes providing tracking and particle identification with $\sim 36$ hits per track.

The SuperLHC upgrade poses tight constraints on the new inner tracker (e.g.: on the innermost $B$-Layer we expect $\sim 0.9 \mathrm{GHz} \mathrm{cm}{ }^{-2}$ particle rate and a fluence of $\sim 3.4 \cdot 10^{16} \mathrm{~cm}^{-2}-$ or $2.0 \cdot 10^{16} \mathrm{n}_{\mathrm{eq}} \mathrm{cm}^{-2}$ - for a run of $3000 \mathrm{fb}^{-1}$ ). A design entirely based on silicon sensors is under development. The current baseline design includes four layers of pixel detectors and five double layers of silicon strips, with an acceptance extending to $|\eta|<2.5$. Different sensor options are been considered, including planar and 3D Silicon sensors, Diamond sensors and gas-pixel detectors (the Gossip R\&D project [4], that adds a silicon pixel anode/read-out plane to a micromegas chamber detector).

\subsection{Calorimeters}

In general, the electromagnetic and hadronic calorimeters of ATLAS are expected to stand the rates of SuperLHC without loss of performance. The only concern is for the forward calorimeter, a liquid Argon detector covering $3.1<|\eta|<4.9$, which will suffer from the rate because of build-up of space charge from positive ions, and power dissipation (stability against boiling). Two upgrade options are being considered: a) replacement with a detector with a modified geometry of the ionization cell, or b) insertion of an additional warm calorimeter in the space available upstream of the existing forward calorimeter. The latter option would presumably be based on a copper/tungsten - diamond sampling design, and has the advantage of not requiring opening the cryostat that contains the forward and end-cap calorimeters.

In addition, the upgrade of the calorimeters read-out electronics is being considered, in order to increase its radiation hardness, the bandwidth and the triggering capabilities.

\section{The Muon spectrometer}

The background rate in the Muon chambers is dominated by photons and neutrons in the energy range of 0.1 to a few $\mathrm{MeV}$. At $1 \cdot 10^{34} \mathrm{~cm}^{-2} \mathrm{~s}^{-1}$ the interaction rate in the Muon chambers is estimated in the range of $\sim 10$ to $\sim 400 \mathrm{~Hz} \mathrm{~cm}^{-2}$. The estimate suffers from uncertainties in the model and from the large energy dependence of the interaction cross section, and the system has been designed with a safety factor of 5 in the hit rate. The extent of the upgrade required for running at $\sim 1 \cdot 10^{35} \mathrm{~cm}^{-2} \mathrm{~s}^{-1}$ will become clear only with the first run of LHC, but it is very likely that at least the inner station of the end-cap spectrometer will require upgrades in order to increase the range of sustainable particle rate, to reduce the read-out bandwidth, and possibly to improve the performance of the Muon Level-1 trigger.

Several technologies are being considered for the upgrades of the Muon chambers. Besides the upgrade for phase-2, an upgrade for phase-1 is also being considered, which would be limited to addition of a detector in the inner end-cap station, in order to increase the tracking ability in region $2<|\eta|<2.7$. The different technologies under study are presented in the following sections. 


\subsection{Small-tube precision chambers}

The Monitored Drift Tube chambers are used as precision chambers in all stations of the Muon spectrometer. They are formed by aluminium drift tubes, $30 \mathrm{~mm}$ diameter, operated at 3 bar with an $\mathrm{Ar}-\mathrm{CO}_{2}$ gas mixture. A chamber, formed by 6 or 8 layers of tubes, is expected to measure a track with an accuracy of about $50 \mu \mathrm{m}$. A detector based on small-tubes with $15 \mathrm{~mm}$ diameter [5] has been proposed for the upgrade of the Muon spectrometer. Compared to the current design, the smaller radius tubes would have a channel occupancy reduced by a factor $1 / 2$, the charge collection time reduced by a factor $1 / 3$ (due to higher drift velocity near the anode), and the effects of space charge (positive ions) reduced by a factor of about $1 / 8$.

\subsection{Upgraded TGCs}

Thin Gap Chambers are used in the end-cap spectrometer for triggering and for providing tracking also in the $2^{\text {nd }}$ (non-bending) coordinate. An upgraded version of this technology [6] can provide: a) precision tracking by analogue read-out of strips orthogonal to the anode wires; b) second coordinate by grouping of anode wires or pad read-out; c) higher rate capability by reduced surface resistivity (10-20 $\mathrm{kOhm} / \mathrm{square})$. In addition, the upgraded TGC might be used as triggering devices, as in the current spectrometer.

\subsection{Micromegas}

Micromegas chambers are suited for tracking at high rate, and their resolution in both coordinates can be optimized with proper pitch and segmentation of the read-out strips. The ATLAS R\&D effort [7] focuses on: a) optimization of the performance for tracks with incidence angle between 90 and 45 degrees; b) the development of construction methods for relatively large detectors $\left(\sim 1.1 \mathrm{~m}^{2}\right)$; c) the use of a gas mixture that would facilitate the integration in the ATLAS Muon spectrometer (such as $\mathrm{Ar}-\mathrm{CO}_{2}$ mixtures).

\section{References}

[1] F. Zimmermann, CERN upgrade plans for the LHC and its injectors, these proceedings.

[2] N. Hartman, private communication (see: http://indico.cern.ch/getFile.py/access? contribId=25\&sessionId=5\&resId=1\&materialId=slides\&con

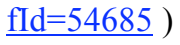

[3] C. Da Via, private communication (see also: http://indico.cern.ch/materialDisplay.py?sessionId=3\&materialId $=0 \&$ confId $=45460$ )

[4] H. van der Graaf, private communication (see also: A. Romaniouk et al., ATL-UPGRADE-SLIDE2009-141, http://cdsweb.cern.ch/record/1180841? $\mathrm{ln}=$ en)

[5] H. Kroha and R. Richter, private communication (see also: http://indico.cern.ch/materialDisplay.py?contribId=1\&materialId=slides\&confId=43987 )

[6] G. Mikenberg, private communication (see also: http://indico.cern.ch/materialDisplay.py?contribId=4\&materialId=slides\&confId=43987 )

[7] J. Wotschack, private communication (see also: P. Iengo et al., ATL-UPGRADE-SLIDE-2009-149, http://cdsweb.cern.ch/record/1183853?!n=en ) 